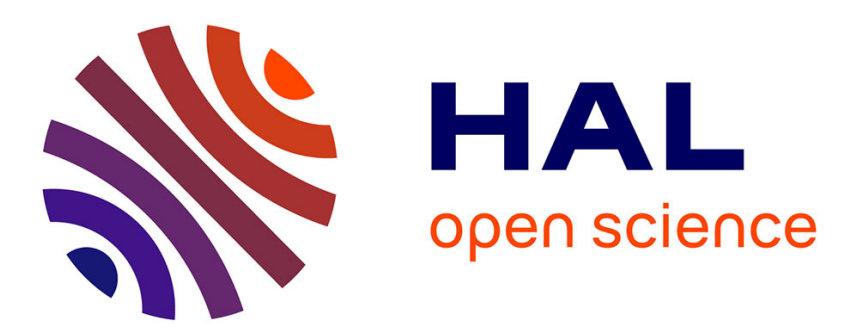

\title{
Effect of non-uniform flexibility on hydrodynamic performance of pitching propulsors
}

\author{
Samane Zeyghami, Keith W Moored
}

\section{To cite this version:}

Samane Zeyghami, Keith W Moored. Effect of non-uniform flexibility on hydrodynamic performance of pitching propulsors. International Symposium on Transport Phenomena and Dynamics of Rotating Machinery, Dec 2017, Maui, United States. hal-02369258

\section{HAL Id: hal-02369258 \\ https://hal.science/hal-02369258}

Submitted on 18 Nov 2019

HAL is a multi-disciplinary open access archive for the deposit and dissemination of scientific research documents, whether they are published or not. The documents may come from teaching and research institutions in France or abroad, or from public or private research centers.
L'archive ouverte pluridisciplinaire HAL, est destinée au dépôt et à la diffusion de documents scientifiques de niveau recherche, publiés ou non, émanant des établissements d'enseignement et de recherche français ou étrangers, des laboratoires publics ou privés. 


\title{
Effect of non-uniform flexibility on hydrodynamic performance of pitching propulsors
}

\author{
Samane Zeyghami ${ }^{1 *}$, Keith W. Moored ${ }^{1}$
}

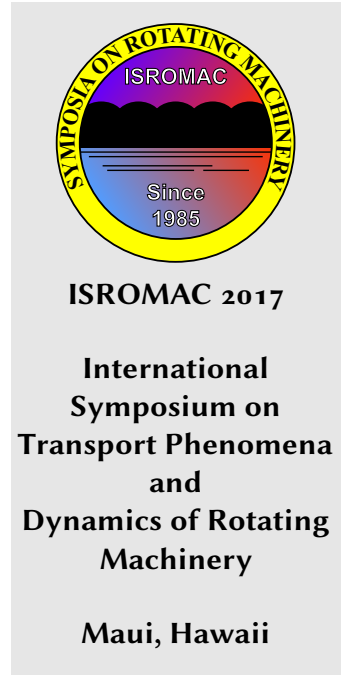

December 16-21, 2017

\begin{abstract}
Many aquatic animals propel themselves efficiently through water by oscillating flexible fins. These fins are, however, not homogeneously flexible, but instead their flexural stiffness varies along their chord and span. Here, we developed a low order model of these functionally-graded materials where the chordwise flexibility of the foil is modeled by one or two torsional springs along the chordline. The torsional spring structural model is then strongly coupled to a boundary element fluid model to simulate the fluid-structure interactions. We show that the effective flexibility of the combined fluid-structure system scales with the ratio of the added mass forces acting on the passive portion of the foil and the elastic forces defined by the torsional spring hinge. We further detail the dependency of the propulsive performance on the flexibility and location of the single torsional spring for a foil that is actively pitching about its leading edge. Our results show that increasing the flexion ratio by moving the spring away from the leading edge leads to enhanced propulsive efficiency, but compromises the thrust production. Proper combination of two flexible hinges, however, can result in a gain in both the thrust production and propulsive efficiency.

Keywords

Nonuniform flexibility - Unsteady propulsion - Propulsive performance - Pitching - Bending patterns

'Department of Mechanical Engineering and Mechanics, Lehigh University, Bethlehem, USA

${ }^{*}$ Corresponding author: saz316@lehigh.edu
\end{abstract}

\section{NOMENCLATURE}

$\begin{array}{ll}f & =\text { pitching frequency } \\ \rho & =\text { fluid density } \\ c & =\text { fin chord } \\ A & =\text { trailing edge amplitude) } \\ \lambda & =\text { flexion ratio } \\ U & =\text { swimming velocity) } \\ k & =\text { reduced frequency } \\ \Pi_{k} & =\text { effective stiffness } \\ I & =\text { matrix of moment of inertia } \\ K & =\text { stiffness matrix } \\ C & =\text { damping matrix } \\ C_{t}, C_{t}^{\prime} & =\text { thrust coefficient } \\ C_{p}, C_{p}^{\prime} & =\text { power coefficient } \\ N_{i} & =\text { inertial moment } \\ N_{f} & =\text { hydrodynamic moment } \\ N_{h} & =\text { hinge moment }\end{array}$

\section{INTRODUCTION}

Flying and swimming animals propel themselves rapidly and efficiently through a fluid using flexible propulsors. A substantial line of work has already confirmed that flexible propulsors are advantageous to rigid ones in aquatic locomotion, specifically with regard to propulsive efficiency $[1,2,3,4]$. Some have argued that the interactions between the fluid and the structure deforms the foil in the direction of the fluid. These deformations lead to curvature-induced thrust increases [5] as well as a favorable phase lag between the pitching and heaving motions of the foil which in return enhances propulsive efficiency $[6,7]$. In addition, the occurrence of resonance is argued to play an important role in enhancing propulsive performance of flexible foils. Previous studies have shown that the efficiency is maximized at or near the resonance frequency of the combined fluid-structure system $[8,4]$. The resonance frequency of the combined system is a function of the inertial properties of the structure as well as the added mass arising from inertia of the fluid. However, when the flexibility is variable along the chord, the scaling of the resonance frequency of the fluid-structure system is non-trivial, a topic that we will attend to in the present study.

The propulsive appendages of swimming and flying animals are made of functionally-graded materials where the flexibility varies both along the chord and span. In fact, Combes and Daniel [9] measured the flexural stiffness of several insect wings and found that it declines sharply from the wing base to wing tip, in the spanwise direction, and from the leading edge to the trailing edge, in the chordwise direction. Similarly, the flexibility of the propulsive surfaces of swimming animals (such as fluke, fin and tail) appear to be non-uniform and decline from the leading to trailing edge and from the center to the edges $[10,11,12]$. Inspired by these observations, a number of recent studies have suggested that the distribution of the flexibility along the foil, in addition to its overall flexibility, may play an important role in enhancing the propulsive performance. Comparing different distributions of flexibility along a two dimensional thin 
foil undergoing small amplitude heaving motions, Moore [13] has suggested that the concentration of the flexibility at the leading edge enhances thrust production. In another study, Riggs et al. [14] have tested the thrust production of a flexible fin with a standard NACAoo12 cross-sectional shape alongside fins with a stiffness profiles mimicking that of a Pumpkinseed Sunfish. They showed that bio-mimetic fins generate more thrust regardless of the overall stiffness of the fin, showing that the performance improvement is due to the stiffness profile itself and not the flexibility alone. Similar conclusions were found by [12] in an experimental study on the propulsive performance of robotic fins with variable chordwise flexibility. They found that fins with variable flexibility outperformed the fins with uniform flexibility with regard to both thrust production and propulsive efficiency.

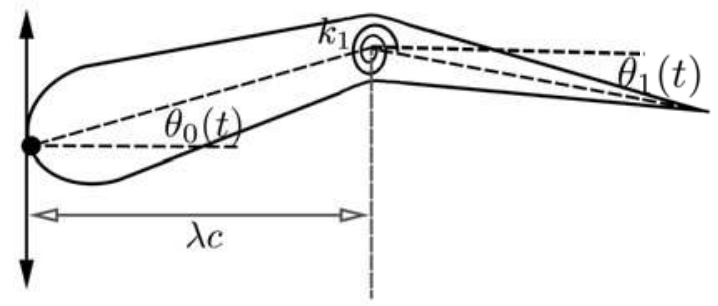

Figure 1. Schematic of the model for a single spring.

Here we aim to probe the effect of the distribution of flexibility on the propulsive performance of a pitching foil by separating the effect of the overall flexibility of a pitching foil from that of its bending pattern. We model the chordwise flexibility of the foil with a series of torsional springs with varying flexibility. The effect of the bending patterns is modeled via changing the location of the spring along the chord. First, we investigate the propulsive performance of a pitching foil with a single flexible joint. We detail the effect of both the overall flexibility and the bending pattern of the foil. Next, we repeat our numerical experiment on a pitching foil with two flexible joints. This time the location of the flexible joints are fixed but the distribution of the flexibility is varied. This study is a primary step toward understanding the role the functionally graded materials on the propulsive performance. Results of this study can also inspire design of innovative and non-traditional propulsors.

\section{PROBLEM DEFINITION}

All the numerical experiments are performed on a two dimensional foil where the leading edge of the foil is actively pitching with a peak-to-peak amplitude of $2 \theta_{0}=10^{\circ}$. There are either one or two flexible joints along the chord modeled by torsional springs (figure 1). The distance of the flexible joint from the leading edge, normalized by the chord length, is quantified by the flexion ratio, $\lambda$. The flapping frequency and swimming velocity are kept constant across these simulations at $2.87 \mathrm{~Hz}$ and $0.1 \mathrm{~m} / \mathrm{s}$ resulting in a reduced frequency of $k=2.87$ (defined as $k=\frac{f c}{U}$ ) and a Strouhal number (de- fined as $S t=\frac{f A}{U}$ ) of 0.5. This $S t$ is defined for a rigid foil without a flexible joint. However, the real $S t$ of the flow is an output of the system and varies with the trailing edge amplitude, which itself is a function of the flexibility and flexion ratio.

For materials with similar densities as that of the surrounding fluid (in the present study $\rho_{s}=\rho$ ), the flexibility of the combined fluid-structure system is a function of the added mass forces of the fluid and the elastic forces of the structure. We define $\Pi_{k}$ as the ratio of these forces which characterizes the effective flexibility of the combined fluidstructure system.

$$
\Pi_{k}=\left(1-\lambda^{2}\right) \sqrt{\frac{\rho b c^{4} f^{2}}{k}}
$$

where $\rho, f, c$, and $k$ respectively are the fluid density, pitching frequency, chord length, and the spring stiffness. The numerator is the added mass force represented as a cylinder of fluid with a diameter equal to length of the passive portion of the foil multiplied by a characteristic acceleration. When only added mass forces are modeled, $\Pi_{k}$ is directly proportional to the ratio of the driving and resonance frequencies.

Both flexibility and flexion ratio are changed and their effect on the propulsive performance is detailed. The performance is analyzed using thrust and power coefficients as well the propulsive efficiency which are defined below.

$$
C_{t}=\frac{T}{0.5 \rho U^{2} b c}, \quad C_{p}=\frac{P}{0.5 \rho U^{3} b c}
$$

where $T$, and $P$ are the thrust and input power. $b$ is the span length that is set to 1 . Alternatively, we normalized thrust and power with trailing edge velocity as defined in eqn. 3 . Note that the trailing edge amplitude is an output of a flexible foil system.

$$
C_{t}{ }^{\prime}=\frac{T}{0.5 \rho A^{2} f^{2} b c}, \quad C_{p}{ }^{\prime}=\frac{P}{0.5 \rho U A^{2} f^{2} b c}
$$

where $A$ is the trailing edge amplitude.

\section{NUMERICAL METHODS}

The flow over the foil is modeled using a two-dimensional potential flow method in which the flow is assumed to be irrotational, incompressible and inviscid. We follow [15] and [16], in that the general solution to the potential flow problem is reduced to finding a distribution of doublets and sources on the foil surface and in the wake that satisfies no flux boundary condition on the body at each time step. Constant strength source and doublet line elements are distributed over the body and the wake. Each body boundary element is assigned a collocation point which is shifted a small distance under the body surface (here $1 \%$ of the local thickness of the body). The constant potential Dirichlet condition is enforced at the collocation points to ensure a no flux boundary condition on the body surface. Additionally, at each time step a wake boundary element is shed with a strength that is set by applying an 
explicit Kutta condition, where the vorticity at the trailing edge is set to zero $[17,18,19]$. A wake rollup algorithm is employed to ensure that the wake does not support any force. The wake elements advect by the local velocity at the wake panel edge points. During the wake rollup the point vortices, representing the ends of the wake doublet elements, must be de-singularized for numerical stability of the solution [20]. To do so, at a small cutoff radius of $\epsilon=0.05 c$, the irrotational induced velocities from the point vortices are replaced by a rotational Rankine core model. The tangential perturbation velocity component is calculated by local differentiation of the perturbation potential. Finally, the pressure acting on the body is found via applying the unsteady Bernoulli equation. More details can be found in $[15,21,22]$.

The structural flexibility is modeled via torsional springs which connect the structural mesh elements together. The kinematics of the leading structural element is always prescribed. Equation 4 governs the dynamics of the passive structural elements.

$$
I \ddot{\Theta}+C \dot{\Theta}+K \Theta=N_{f}+N_{i}+N_{h}
$$

where $N_{f}$ is the hydrodynamic moment exerted about the joint location. $N_{i}$ is the inertial moment due to the translational velocity of the center of mass of the corresponding element. $N_{h}$ is the moment exerted by the forces at the joint which keep the elements together. $I$ is the matrix of moments of inertia about the joint points. $K$ and $C$ are the matrices of the structural stiffness and damping, respectively. $\Theta$ is a vector containing the orientation of the passive elements. For a foil with two flexible joints we have:

$I \equiv\left[\begin{array}{cc}I_{1} & 0 \\ 0 & I_{2}\end{array}\right] \quad K \equiv\left[\begin{array}{cc}k_{1}+k_{2} & -k_{2} \\ -k_{1} & k_{2}\end{array}\right] \quad C \equiv\left[\begin{array}{cc}c_{1}+c_{2} & -c_{2} \\ -c_{1} & c_{2}\end{array}\right]$

where $I_{i}$ is the moment of inertia of the $i^{t h}$ element. $c_{i}$ and $k_{i}$ are the structural damping and the stiffness of the spring attached to the leading edge of the $i^{\text {th }}$ element.

To solve the fluid-structure interaction problem, equation 4 is discritized in time, using the trapezoidal rule (equations 6 and 7), and solved within each small time step via a strong coupling between the fluid and structural solvers which is accelerated by the Aitken method. To improve the convergence properties of the solver, while keeping its efficiency, we use two different time step sizes $\Delta t$ and $\Delta t_{s}$ for the fluid and structure solvers, respectively, where $\Delta t_{s}=\frac{\Delta t}{N_{s}}$. $N_{s}$ is set to 100 in the present simulations.

$$
\begin{aligned}
& \hat{\Theta}^{m+1}=\hat{\Theta}^{m}+\frac{1}{2}\left(\dot{\hat{\Theta}}^{m}+\dot{\hat{\Theta}}^{m+1}\right) \Delta t_{s} \\
& \dot{\hat{\Theta}}^{m+1}=\dot{\hat{\Theta}}^{m}+\frac{1}{2}\left(\ddot{\hat{\Theta}}^{m}+\ddot{\hat{\Theta}}^{m+1}\right) \Delta t_{s}
\end{aligned}
$$

where superscripts $m$ and $m+1$ represent the values at times $t_{s}^{m}$ and $t_{S}^{m+1}$, respectively. $\hat{x}$ represents any variable $x$ within the structure solver. Substituting equation 7 into equation 6 and solving for $\ddot{\Theta}^{m+1}$ we get:

$$
\ddot{\Theta}^{m+1}=\left(\frac{2}{\Delta t_{s}}\right)^{2}\left(\hat{\Theta}^{m+1}-\hat{\Theta}^{m}\right)-\left(\frac{4}{\Delta t_{s}}\right) \dot{\hat{\Theta}}^{m}-\ddot{\Theta}^{m}
$$

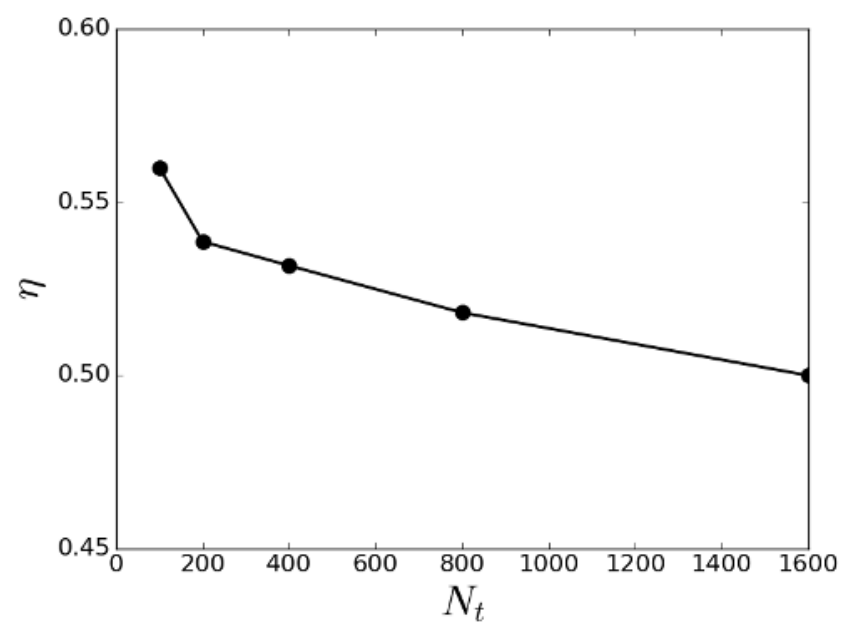

(a)

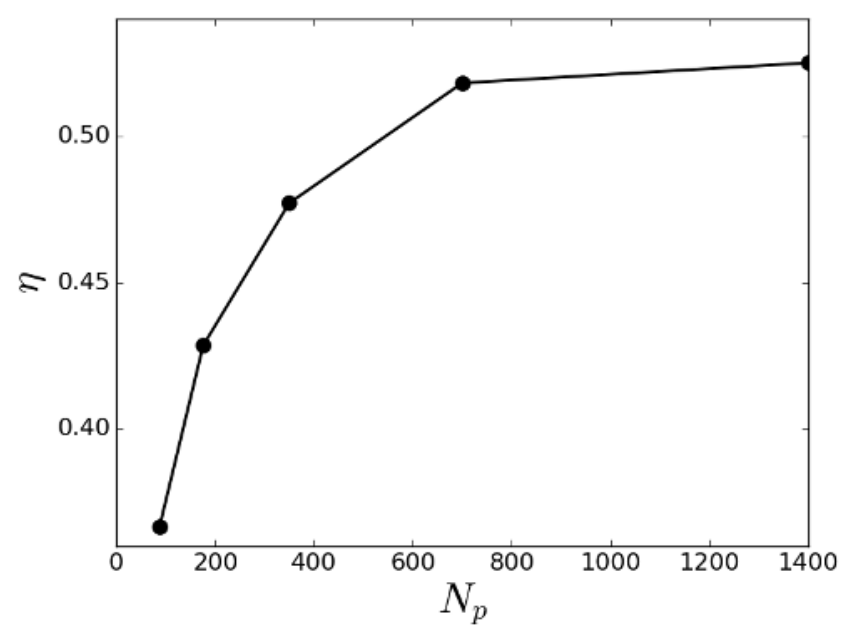

(b)

Figure 2. Propulsive efficiency as a function of (a) number of time steps and (b) number of body panels.

Similarly, equation 6 can be rearranged to get an expression for $\dot{\hat{\Theta}}^{m+1}$ as a function of $\hat{\Theta}$ :

$$
\dot{\hat{\Theta}}^{m+1}=\frac{2}{\Delta t_{s}}\left(\hat{\Theta}^{m+1}-\hat{\Theta}^{m}\right)-\dot{\hat{\Theta}}^{m}
$$

Where the right hand sides of both equations 8 and 9 are known from the previous iteration.

Substituting equations 9 and 8 into 4 , we can rewrite the governing equations as a linear, but coupled, system of equations as follows: 

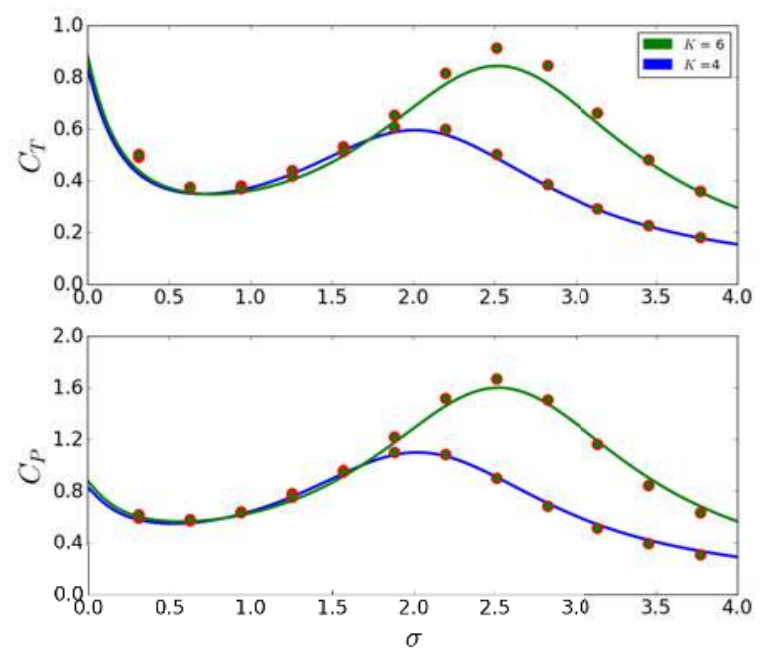

Figure 3. Analytical solutions for thrust and power coefficient as a function of reduced frequency, for two different non-dimensional spring stiffnesses, are shown with solid lines. These solutions are taken from [23]. Closed circles are the solutions calculated by the present numerical method.

$$
\begin{aligned}
& A \hat{\Theta}^{m+1}=\hat{b}^{m} \\
& A=K+\left(\frac{2}{\Delta t_{s}}\right)^{2} I+\left(\frac{2}{\Delta t_{s}}\right) C \\
& \hat{b}^{m}=\left(\frac{2}{\Delta t_{s}}\right)^{2} I \hat{\Theta}^{m}+\left(\frac{4}{\Delta t_{s}}\right) I \dot{\hat{\Theta}}^{m}+I \ddot{\hat{\Theta}}^{m}+\left(\frac{2}{\Delta t_{s}}\right) C \hat{\Theta}^{m} \\
& +C \dot{\hat{\Theta}}^{m}+\hat{N}_{i}^{m}+\hat{N}_{f}^{m}+\hat{N}_{h}^{m}
\end{aligned}
$$

Equation 10 together with 6 and 7 form a complete set of equations for the structure. The set of structure equations are first initialized by the known solution from the previous time step (of the fluid's solver) and then iterated $N_{s}$ times to advance the solution as much as $\Delta t$.

To improve convergence, equation 10 is uncoupled by employing a Gauss-Seidel formulation where the newly obtained solution for the orientation of the first element is used to obtain the solution for the second element at each time step $t_{s}$.

The Aitken acceleration method is commonly used in the numerical simulation of fluid-structure interactions and is proven to be sufficiently simple and efficient $[24,25,26]$. This method uses the values from the two previous iterations to correct the new solution. We employ Aitken's method to advance the solution in the fluid's solver based on the residual calculated in the previous two iterations. The residual is calculated as the difference in the solution obtained in the structure and the fluid solvers, $\vec{r}_{i}=\hat{\Theta}_{i}-\Theta_{i}$, where $\Theta_{i}$ is a vector representing the orientation of the neutral axis of the foil in the fluid solver.

The solution to the coupled fluid-structure system at each time step $t_{n}=n \Delta t$ is obtained by following the algorithm below:

1. $i=0, r_{0}=1, \tilde{\Theta}_{0}=\Theta_{n-1}, \dot{\tilde{\Theta}}_{0}=\dot{\Theta}_{n-1}, \ddot{\tilde{\Theta}}_{0}=\ddot{\Theta}_{n-1}$, and $\omega_{0}=1 e-2$

2. While $\left\|r_{i}\right\|>\delta$

(a) $i=i+1$

(b) if $i>1$ modify the solution; $\tilde{\Theta}_{i}=\tilde{\Theta}_{i-1}+\omega_{i-1} r_{i-1}$

(c) Calculate the location of the neutral axis of the foil in the fluid solver via known values of the leading element and passive elements.

(d) Calculate the position and velocity of the fluid panels on the foil surface.

(e) Calculate fluid forces and moments.

(f) Solve the solid deformations; $\hat{\Theta}_{i}, \dot{\hat{\Theta}}_{i}$, and $\ddot{\Theta}_{i}$ using equations 10,8 , and 7

(g) Calculate the residual, $r_{i}=\hat{\Theta}_{i}-\tilde{\Theta}_{i}$

(h) Calculate Aitken Acceleration factor; if $i<3, \omega_{i}=\omega_{0}$
else, $\omega_{i}=\omega_{i-1} \frac{\vec{r}_{i-1}\left(\vec{r}_{i-1}-\vec{r}_{i}\right)}{\left\|r_{i-1}-r_{i}\right\|^{2}}$

3. Update the solution for time $t_{n} ; \Theta_{n}=\tilde{\Theta}_{i}, \dot{\Theta}_{n}=\dot{\tilde{\Theta}}_{i}$, and $\ddot{\Theta}_{n}=\ddot{\tilde{\Theta}}_{i}$

where $\delta$ is set to $10^{-8}$. When the solution converges within the $n^{\text {th }}$ time step, we set $n=n+1$ and repeat the steps above to solve for the next time step.

\subsection{Discretization independence}

Figure 2 shows propulsive efficiency as a function of number time steps within an oscillation period, $N_{t}$, and number of body panels, $N_{p}$. The leading edge kinematics and the $S t$ are set to the same values as the main case studies. $\lambda$ and $\Pi_{k}$ are set to 0.8 and 0.3 respectively. $\Pi_{k}=0.3$ marks the resonance frequency of the flow-structure system. It is evident that $\eta$ converges to the discretization independent solutions as the number of body panels and time steps increases. This performance matrices change less than $4 \%$ when $N_{\text {panel }}=$ 700 and $N_{\text {time }}=800$.

\subsection{Validation}

We tested the accuracy of our numerical model by comparing our results against the analytical results presented in [23] for a two dimensional thin foil with a torsional spring at the leading edge. A small amplitude (harmonic) heaving motion is enforced at the leading edge. The foil passively pitches about the leading edge due to the action of fluid, inertial, and elastic forces. We compared both the cycle-averaged thrust and the cycle-averaged power with the analytical solution. Results are shown in Figure 3. 


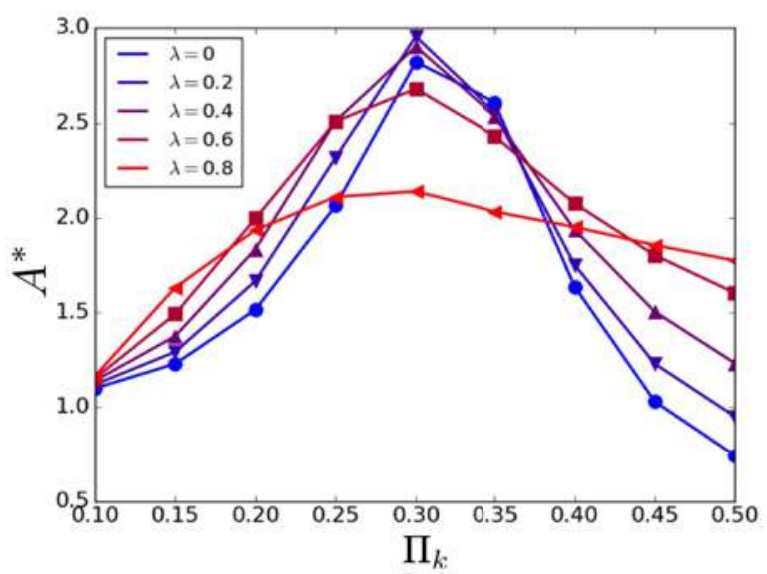

(a)

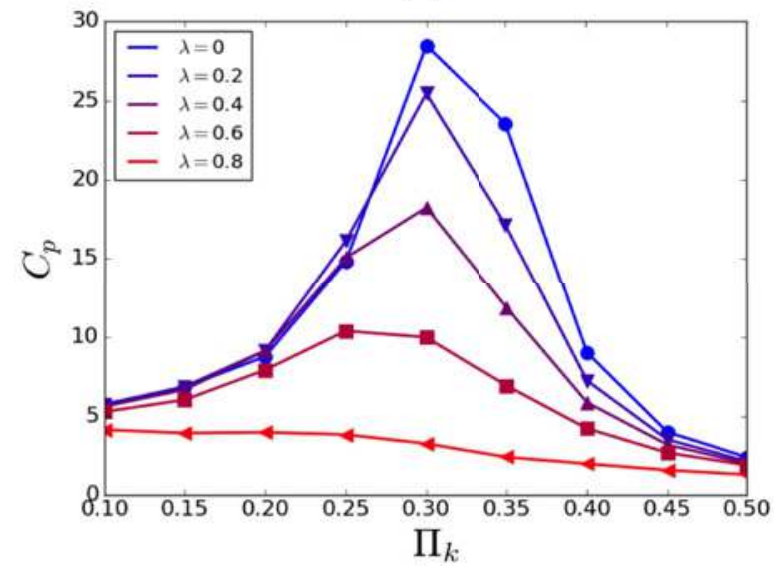

(c)

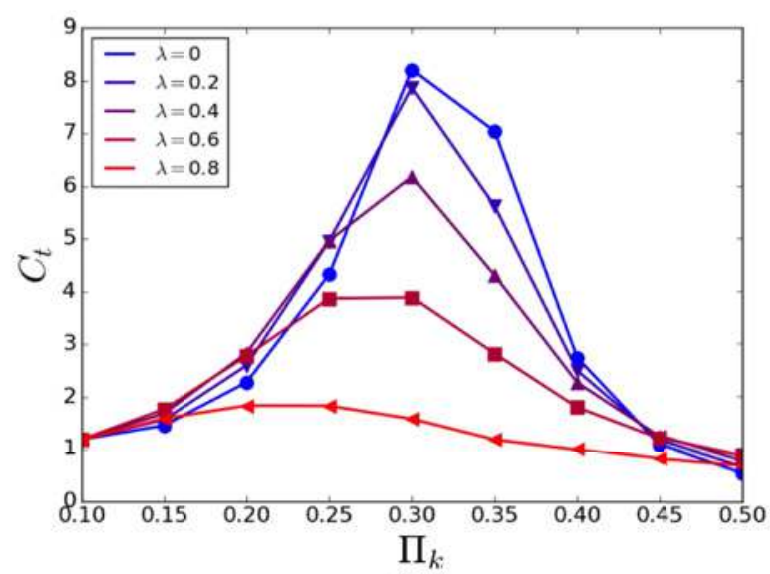

(b)

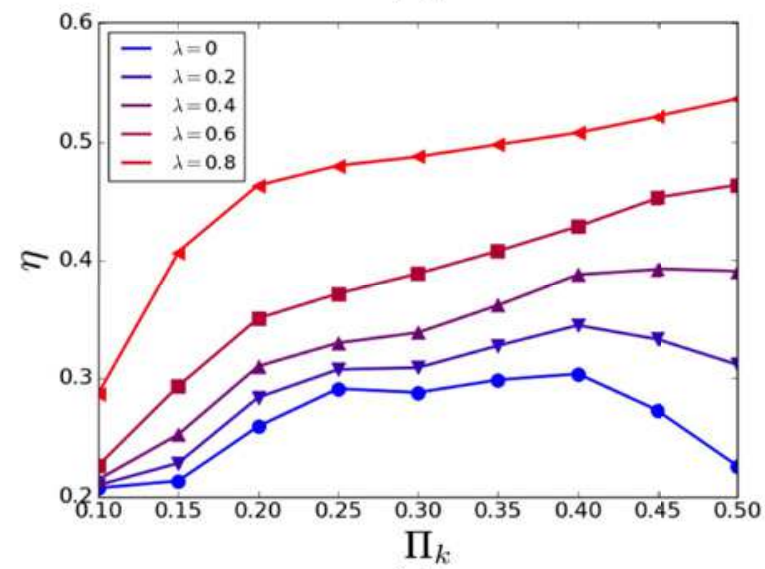

(d)

Figure 4. (a) Trailing edge amplitude, (b) thrust coefficient, (c) power coefficient, and (d) efficiency as a function of $\Pi_{k}$.

\section{RESULTS AND DISCUSSION}

\subsection{Single flexible joint}

Figure 4 a shows the non-dimensional trailing edge amplitude, $A^{*}=\frac{A}{A_{\text {rigid }}}$, as function of effective flexibility for five different flexion ratios. The trailing edge amplitude is maximum at the resonance which occurs at $\Pi=0.3$ for all flexion ratios. The coincidence of the resonance frequency for all $\lambda$ 's shows that the proposed scaling for the effective flexibility appropriately accounts for the effect of the flexion ratio. However, inspecting the trend of variations in $A^{*}$ with $\Pi_{k}$ reveals that the effective damping of the combined fluid-structure system increases for larger $\lambda$ values. It is also worth noting that before and after resonance, higher flexion ratio foils generally experience larger trailing edge amplitude. Around the resonance the relationship is more nonlinear. For flexion ratios smaller than 0.5 the trailing edge amplitude increases with $\lambda$. This relationship is reversed for $\lambda>0.5$.

Figure $4 \mathrm{~b}$ shows variations in thrust and power coefficients as a function of $\Pi_{k}$. Unlike the trailing edge amplitude, the thrust coefficient is generally larger for smaller $\lambda$ 's, except for very rigid foils where increasing $\lambda$ up to 0.5 results in gain in thrust. Inspecting figure $4 \mathrm{c}$ shows that this gain in thrust comes with no additional cost with regard to the power consumption. $C_{t}$ rises up to its peak value at the resonance, for small $\lambda$ values, and then drops quickly with further increase in flexibility. This is unlike the behavior of $C_{t}$ for large $\lambda$ values where the thrust plateaus before resonance and then drops with a mild slope when flexibility increases. It is worth noting that for all flexion ratios, $C_{t}$ increases faster than $C_{p}$ until slightly after resonance. This is reflected in Figure $4 \mathrm{~d}$ where we show changes in the propulsive efficiency, defined as $\eta=\frac{C_{t}}{C_{p}}$, as a function of $\Pi_{k}$. Quick drop in thrust production after the resonance for small $\lambda$ values results in a decline in the propulsive efficiency. In contrast, for large flexion ratios, $\eta$ keeps increasing with flexibility for a wide range of flexibilities.

In figure $5 \mathrm{a}-\mathrm{b}$, we plotted $C_{t}^{\prime}$ and $C_{p}^{\prime}$ as the function of $\Pi_{k}$. When we normalized the thrust by the trailing edge velocity, the peak in the force production and power consumption disappeared implying that the peak is an artifact of the amplified trailing edge amplitude. Variations in the trailing edge amplitude are responsible for the major changes in the thrust production for $\lambda=0$. This, however, is not the case for other $\lambda$ values. The fact that the curves for different $\lambda$ values do not collapse on top of one another implies that the bending 


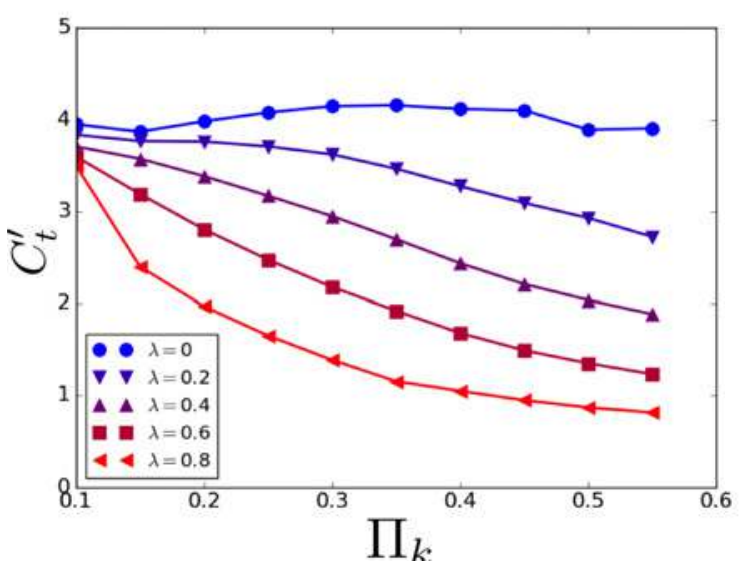

(a)

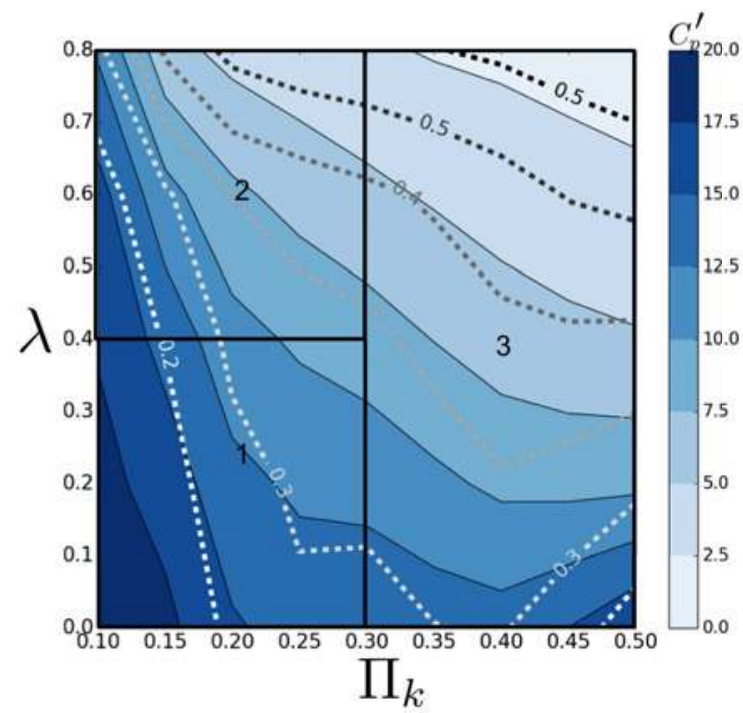

(d)

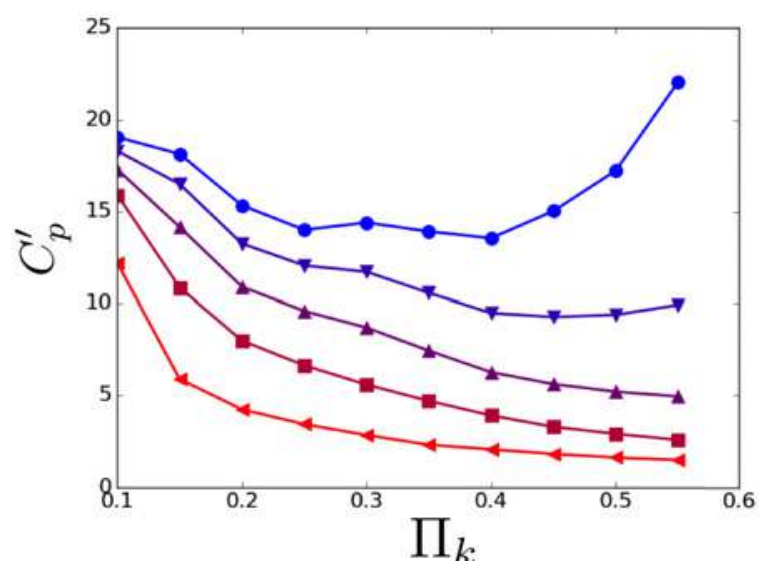

(b)

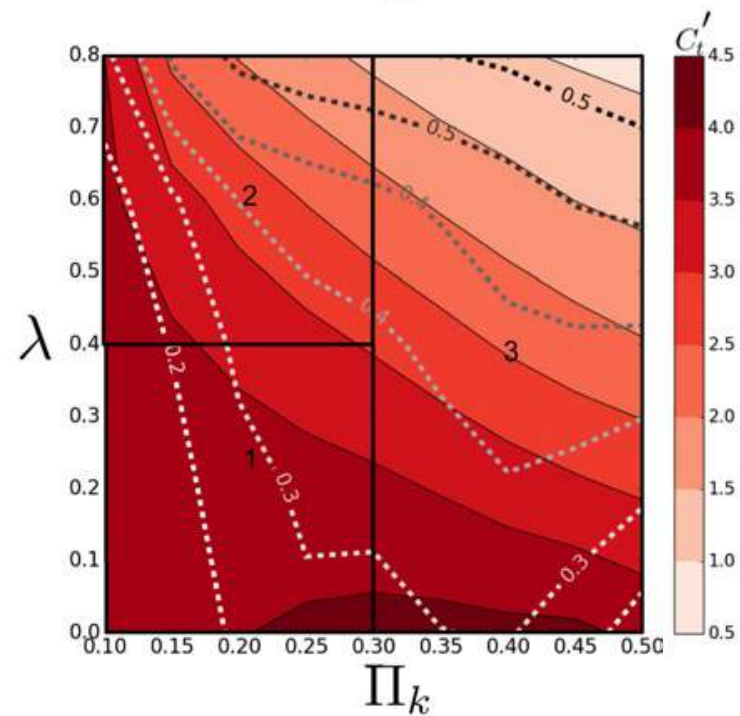

(c)

Figure 5. Variation of thrust (a) and power (b) coefficients, $C_{t}^{\prime}$ and $C_{p}^{\prime}$, defined by equation 3 with $\Pi_{k}$. Contours of $C_{t}^{\prime}$ and $C_{p}^{\prime}$ in $\lambda-\Pi_{k}$ plane. Dotted lines are the contours of propulsive efficiency.

pattern itself, and not only the trailing edge amplitude, play a role in the force production.

The behavior of the $C_{p}^{\prime}$ curves is somewhat different from that of $C_{t}^{\prime}$. Before resonance, the power coefficient appear to drop quickly by increasing flexibility. After the resonance, for small $\lambda$ values, the power coefficient starts to rise again. This results in a drop in efficiency which is due to a simultaneous rise in power consumption and drop in thrust production. For larger $\lambda$ values, however, as flexibility increases, $C_{p}^{\prime}$ keeps declining where the rate of this decline decreases for large flexibilities. Thus, the rate of increase in efficiency with $\Pi_{k}$ (figure 4d) decreases.

To summarize our findings with regard to the propulsive performance of these flexible foils, we plotted contours of $C_{t}^{\prime}$ and $C_{p}^{\prime}$ as a function of $\lambda$ and $\Pi_{k}$ in figure $5^{\mathrm{c}-\mathrm{d} \text {. Contours }}$ of propulsive efficiency are overlayed on both figures with dotted lines. It is worth noting that the contours of efficiency line up with those of power consumption indicating that the propulsive efficiency is mostly governed by the (input) power requirement and not the thrust production. We identified three regions on these contour plots. In region 1, which contains low $\Pi_{k}$ and low $\lambda$ value foils, the thrust coefficient remains relatively constant. The propulsive efficiency can be enhanced by increasing flexibility (contours of propulsive efficiency are almost parallel to the $\lambda$-axis). In region 2 , increasing either flexibility or flexion ratio benefits efficiency but compromises thrust production. In region 3, contours of power consumption, and thus propulsive efficiency, are more or less aligned with the $\Pi_{k}$ axis, meaning that the efficiency is more sensitive to changing the flexion ratio. Thus, implying that for largely flexible foils increasing the flexion ratio can result in improved propulsive performance.

\subsection{Two flexible joints}

In the previous section we showed that changing the bending patterns of a pitching foil via increasing its flexion ratio is the 


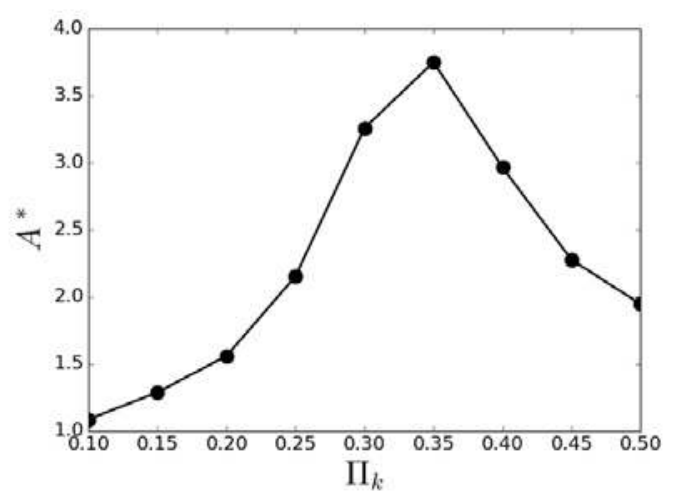

(a)

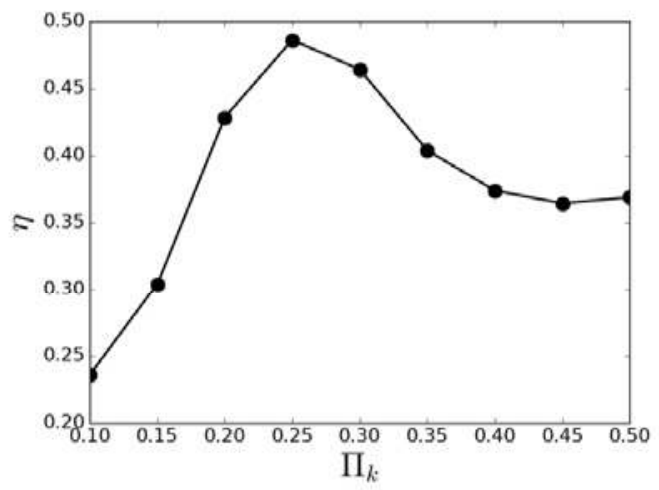

(d)

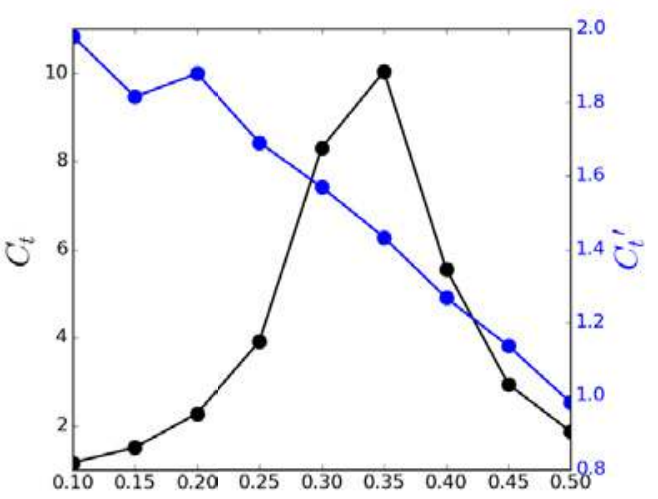

(b)

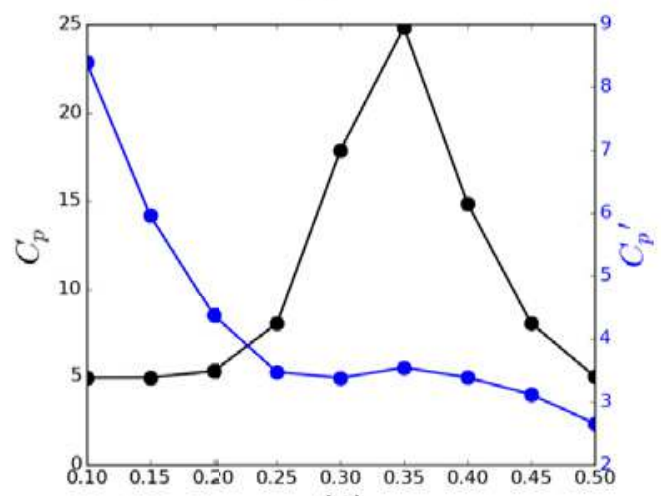

(c)

Figure 6. (a) Trailing edge amplitude, (b) thrust coefficient, (c) power coefficient, and (d) efficiency as a function of $\Pi_{k}$ for two flexible hinge configuration.

key to improving the propulsive efficiency. However, there is a trade off to this gain since smaller flexion ratios are required for larger force production. We hypothesize that combining multiple flexible joints may be the key to gaining both in efficiency and thrust magnitude. To test our hypothesis, we repeated our numerical experiment on a pitching foil with two torsional springs located half a chord away from each other at $\lambda_{1}=0.2$ and $\lambda_{2}=0.7$. The kinematics of the leading edge was kept identical to the cases studied in the previous section.

When multiple flexible joints are allowed not only the flexiblity of the individual joint, but also the profile of flexibility distribution along the chord will affect the foil's deformations and, thus, its propulsive performance. Here, we chose a profile which maintains the effective flexibility of the foil unchanged along the chord. The spring stiffness, thus, declines quadratically with the normalized distance from the leading edge, $k \propto(\lambda-1)^{4}$. To do so, the stiffness of each one of the springs is determined solely based on its distance from the leading edge using equation 1.

Figure 6a shows the trailing edge amplitude as a function of $\Pi_{k}$. A foil with two flexible joints has two resonances, which only one is captured in this figure. The resonance occurs at $\Pi_{k}$ of 0.35 which is slightly larger than the resonance $\Pi_{k}$ for each individual flexible joint. This could be due to increased circulatory effects or existence of nonlinear added mass effects when combining the two joints. In comparison to one flexible joint configurations, the trailing edge amplitude of the present configuration reaches a higher maximum at the resonance, and drops slower afterward. The deformation of the middle element appear to be maximum at the resonance (figure 7). However, the amplitude of the motion of the last element continue to increase after the resonance attenuating the drop in trailing edge amplitude.

In figure $6 \mathrm{~b}$, in black is shown the cycle-averaged thrust coefficients, $C_{t}$. Similar to the results in the previous section, the peak thrust generation occurs at the resonance but the magnitude of the peak is substantially higher. This is partially due to the increased trailing edge amplitude which leads to enhanced added mass forces and a favorable reorientation of the hydrodynamic force relative to the propulsive direction. For $\Pi_{k} \leq 0.3$, the magnitude of $C_{t}$ is similar to that of a foil with a single flexible joint at $\lambda=0.2$ indicating that the effect of the combination of the two joints was not destructive to the thrust production capacity. All the more so,for larger flexibilities, $C_{t}$ is larger than what was achieved by any of the single jointed foil configurations due to the constructive combination effect. In the same figure, in blue is shown $C_{t}^{\prime}$ where thrust is normalized by the trailing edge velocity rather than the swimming velocity. Similar to the case of a 
single spring, $C_{t}^{\prime}$ drops with increasing flexibility.
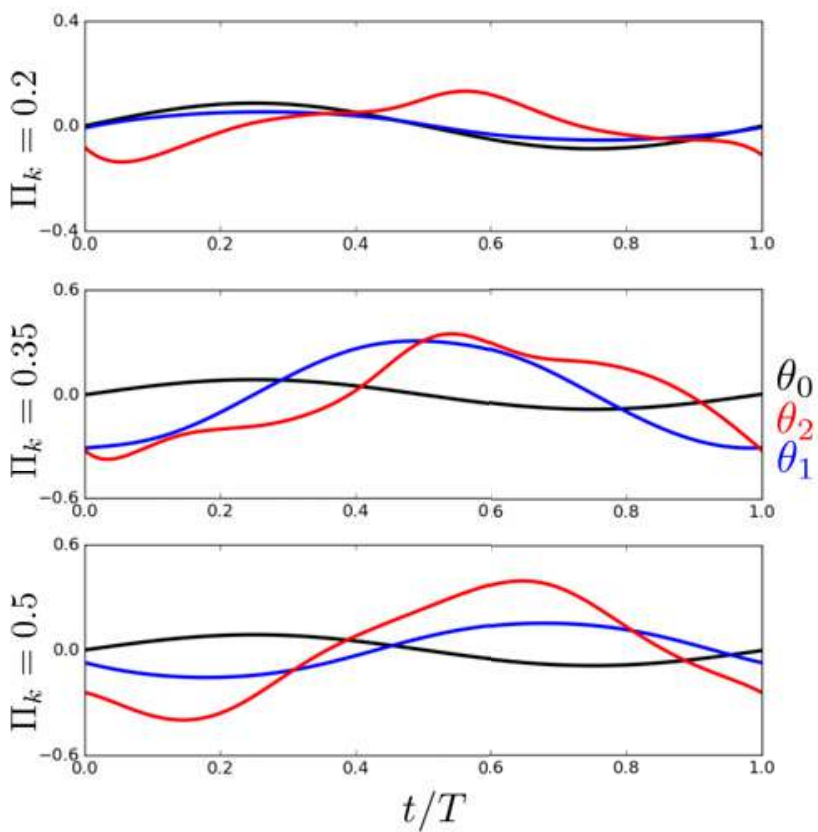

Figure 7. Change in the relative orientation of the solid elements versus time, within one cycle. Black line shows the prescribed pitching motion of the leading edge. Blue and red lines, respectively, show the deflection angles of the second and the third element. The deflection angles are measured relative to the preceding element. The angles are shown for three different $\Pi_{k}$ values of $0.2,0.35$, and 0.5 .

Unlike thrust, the power coefficient, $C_{p}$, remains small for small $\Pi_{k}$ values (figure $6 \mathrm{c}$ ). This results in a quick rise in the propulsive efficiency of the double jointed foil, as reflected in figure $6 \mathrm{~d}$. In the same figure, we show $C_{p}^{\prime}$ in blue. This curve shows a fast drop in the power consumption with flexibility before the resonance (which is typical of high flexion ratio foils in single spring configurations), where at $\Pi_{k} \geq 0.25$ power coefficient is already below what was achieved by most of the single spring configurations (figure 5 ). $C_{p}^{\prime}$ continues to drop even more so after resonance. In such a way, the two spring configuration maintains high propulsive efficiency across a wide range of flexibilities spanning on the both sides of resonance. Overall, the results of this section supports our earlier hypothesis.

\section{CONCLUSIONS}

It has been shown that the unsteady propulsive performance of flexible foils with a single torsional spring hinge is not only a function of their effective flexibility but also their bending patterns. Across all flexibilities tested here, increasing flexion ratio was beneficial to the efficiency while diminishing the thrust production. We showed that the combined effect of the flexibility and the flexion ratio can result in propulsive efficiencies as large as 50\% or more for a purely pitching foil. This is more than $250 \%$ larger than the propulsive efficiency of a rigid foil with the same leading edge kinematics.

Additionally, flexible foils with two flexible joints were examined to probe whether multiple flexible hinges could be used to attenuate the trade-off between thrust and efficiency to achieve fast and efficient swimming simultaneously. The flexibility of the joints was determined such that the effective flexibility was constant along the chord. We found that this combination of flexible joints have a constructive effect on the propulsive performance of a pitching foil with regard to both thrust and efficiency across a wide range of flexibilities.

Finally, it is important to note that the fluid-structure model used in this study is subject to several assumptions. The fluid model, for instance, does not account for viscous effects such as the separation that may occur at the leading edge or along the deforming body, especially when the solid deformation is large. These effects can potentially influence the propulsive performance especially with regard to the power consumption.

\section{ACKNOWLEDGMENTS}

This work was funded by the Office of Naval Research under Program Director Dr B. Brizzolara, MURI Grant Number Nooo 14-14-1-0533.

\section{REFERENCES}

[1] T. Yao-Tsu Wu. Hydromechanics of swimming propulsion. Part 3. Swimming and optimum movements of slender fish with side fins. Fournal of Fluid Mechanics, 46:545-568, 1971.

[2] M. J. Lighthill. Aquatic animal propulsion of high hydromechanical efficiency. Journal of Fluid Mechanics, 44:265-301, 1970.

[3] Peter A Dewey, Birgitt M Boschitsch, Keith W Moored, Howard A Stone, and Alexander J Smits. Scaling laws for the thrust production of flexible pitching panels. Fournal of Fluid Mechanics, 732:29-46, 2013.

[4] Hassan Masoud and Alexander Alexeev. Resonance of flexible flapping wings at low Reynolds number. Physical Review E, 81(5):56304, 2010.

[5] J Katz and D Weihs. Hydrodynamic propulsion by large amplitude oscillation of an airfoil with chordwise flexibility. Journal of Fluid Mechanics, 88(03):485-497, 1978.

[6] Qiang Zhu. Numerical simulation of a flapping foil with chordwise or spanwise flexibility. AIAA journal, 45(10):2448-2457, 2007.

[7] Daniel B Quinn, George V Lauder, and Alexander J Smits. Maximizing the efficiency of a flexible propulsor using experimental optimization. F. Fluid Mech, 767:430-448, 2015.

[8] P. A. Dewey, B. M. Boschitsch, K. W. Moored, H. A. Stone, and A. J. Smits. Scaling laws for the thrust production of flexible pitching panels. Fournal of Fluid Mechanics, 732:29-46, 2013 . 
[9] SA Combes and TL Daniel. Flexural stiffness in insect wings i. scaling and the influence of wing venation. fournal of experimental biology, 206(17):2979-2987, 2003.

[10] FE Fish and GV Lauder. Passive and active flow control by swimming fishes and mammals. Annu. Rev. Fluid Mech., 38:193-224, 2006.

[11] Neil Bose, Jon Lien, and Juha Ahia. Measurements of the bodies and flukes of several cetacean species. Proceedings of the Royal Society of London B: Biological Sciences, 242(1305):163-173, 1990.

[12] AK Kancharala and MK Philen. Optimal chordwise stiffness profiles of self-propelled flapping fins. Bioinspiration \& Biomimetics, 11(5):056016, 2016.

[13] M Nicholas J Moore. Torsional spring is the optimal flexibility arrangement for thrust production of a flapping wing. Physics of Fluids, 27(9):091701, 2015.

[14] Paul Riggs, Adrian Bowyer, and Julian Vincent. Advantages of a biomimetic stiffness profile in pitching flexible fin propulsion. Journal of Bionic Engineering, 7(2):113-119, 2010.

[15] J. Katz and A. Plotkin. Low-speed aerodynamics. Cambridge University Press, New York, NY, second edition, 2001.

[16] D. B. Quinn, K. W. Moored, P. A. Dewey, and A. J. Smits. Unsteady propulsion near a solid boundary. Fournal of Fluid Mechanics, 742:152-170, feb 2014.

[17] David Joe Willis. An Unsteady, Accelerated, High Order Panel Method with Vortex Particle Wakes. Phd, Massachusetts Institute of Technology, 2006.

[18] Seong Yong Wie, Seongkyu Lee, and Duck Joo Lee. Potential panel and time-marching free-wake-coupling analysis for helicopter rotor. Fournal of Aircraft, 46(3):1030-1041, may 2009.

[19] Yulin Pan, Xiaoxia Dong, Qiang Zhu, and Dick K. P. Yue. Boundary-element method for the prediction of performance of flapping foils with leading-edge separation. Journal of Fluid Mechanics, 698:446-467, apr 2012.

[2o] Robert Krasny. Desingularization of Periodic Vortex Sheet Roll-up. Journal of Computational Physics, 65:292313, 1986.

[21] Keith W Moored and Daniel B Quinn. Inviscid scaling laws of a self-propelled pitching airfoil. arXiv preprint arXiv:1703.08225, 2017.

[22] Emre Akoz and Keith W Moored. Unsteady propulsion by an intermittent swimming gait. arXiv preprint arXiv:1703.06185, 2017.

[23] M Nicholas J Moore. Analytical results on the role of flexibility in flapping propulsion. Fournal of Fluid Mechanics, 757:599-612, 2014.

[24] Daniel P Mok and WA Wall. Partitioned analysis schemes for the transient interaction of incompressible flows and nonlinear flexible structures. Trends in computational structural mechanics, Barcelona, 2001.

[25] Ulrich Küttler and Wolfgang A Wall. Fixed-point fluidstructure interaction solvers with dynamic relaxation. Computational Mechanics, 43(1):61-72, 2008.

[26] Iman Borazjani, Liang Ge, and Fotis Sotiropoulos. Curvilinear immersed boundary method for simulating fluid structure interaction with complex $3 \mathrm{~d}$ rigid bodies. Fournal of Computational physics, 227(16):7587-7620, 2008. 